\title{
Psicosis y diferencias sociales: Comparando la prevalen- cia de las psicosis en dos medios urbanos diferenciados.
}

\author{
Psychosis and social differences: Comparing psychosis prevalence between two socially \\ differentiated urban settings.
}

\author{
Jorge L. Tizón ${ }^{\mathrm{a}}$, Josep Ferrando ${ }^{\mathrm{b}}$, Jordi Artigue ${ }^{\mathrm{a}}$, Belén Parra ${ }^{\mathrm{c}}$, Antonia Parés ${ }^{\mathrm{d}}$, Marta Gomàd, \\ Conxita Pérez ${ }^{\mathrm{a}}$, Françesc Pareja ${ }^{\mathrm{d}}$, Marta Sorribes ${ }^{\mathrm{b}}$, Belén Marzarid, Yanet Quijada ${ }^{\mathrm{a}}$, Laia Cataláa \\ ${ }^{a}$ Equipo de Atención Precoz a los Pacientes en riesgo de Psicosis (EAPPP), Institut Catalá de la Salut, Cór- \\ sega 544, bajos, 08025 Barcelona. 'Instituto Municipal de la Salud de Barcelona, Paseig Marítim 25-29, \\ 08003, Barcelona. ${ }^{c}$ Universidad de Barcelona, Departamento de Trabajo Social. ${ }^{d}$ Unidad de Salud Mental \\ de Sant Martí-La Mina, Institut Catalá de la Salut, Plaza de la Infancia s/n, 08020, Barcelona.
}

Correspondencia: Jorge L.Tizón (jtizon@gencat.cat)

RESUMEN: Objetivos: Contribuir a la reflexión sobre la etiología y/o los factores de riesgo para las psicosis comparando la prevalencia en población general y población de riesgo de la esquizofrenia y otras psicosis en dos barrios de Barcelona (España).

Método: Nuestras aportaciones en este trabajo se apoyan sobre todo en un estudio descriptivo transversal de todos los pacientes con psicopatología detectados en la USM de Sant Martí-La Mina: un territorio geodemográfica y asistencialmente delimitado formado por 5 Áreas Básicas de Salud (103.615 habitantes.

Resultados: Sobre un total de 21.536 pacientes con registro de casos abierto desde el año 1982 hasta el año 2000, se halló que 838 cumplían los criterios restrictivos para ser diagnosticados como "esquizofrénicos" $(\mathrm{N}=476)$ o "afectados por otras psicosis" $(\mathrm{N}=362)$. Sin embargo, las prevalencias de esquizofrenia y otras psicosis en el barrio sujeto a más factores de riesgo psicosociales eran alrededor de 2 veces mayores que las encontradas en el barrio colindante por el mismo equipo y en el mismo período temporal.

Conclusión: Es necesario tener en cuenta el gran peso de los factores de sociales y psicosociales para poderse explicar las diferencias de prevalencia de la esquizofrenia y otras psicosis entre diferentes poblaciones.

PALABRAS CLAVE: Esquizofrenia, genética, psicosis, factores de riesgo sociales, factores de riesgo psicológicos, ciudades, barrios.
ABSTRACT: Objectives: To contribute to the discussion about aetiology and risk factors of psychosis comparing the prevalence in general population and in "age of risk population" for schizophrenias and other psychoses on two neighbourhoods of Barcelona (Spain).

Method: We base our reflections in a transversal study about the results of an informatized case register of all the patients' with detected psychopathology in this geodemographic and assistencially differentiated area: 5 Basic Areas of Health (103.615 inhabitants).

Results: The total "psychopathological patients" detected were 21.536. From them, 838 completed the restrictive criteria to be diagnosed as "schizophrenics " (476) or "affected by other psychoses" (362). Among the neighborhood charged with psychosocial risk factors and the other 4 adjacent basic areas of health, assisted for the same team, so much clinical as investigator, the incidence and the prevalence of the schizophrenia and other psychoses is twice as much, almost in each group diagnosis.

Conclusion: It seems necessary to keep in mind the great weight of the social and psychosocial factors to explain those differences of incidence and prevalence of the schizophrenia and the psychoses in different populations.

KEY WORDS: Schizophrenia, genetics, psychoses, social risk factors, psychological risk factors, urbanicity, cities.

La realización de este trabajo ha sido apoyada por las becas para el Proyecto SASPE (02E/99 de la Fundació Seny tras evaluación de la Agencia para la Evaluación de la Investigación y las Tecnologías Médicas de Catalunya) y el Proyecto LISMEP (Ayuda MaratóTV 02 / 0133 de la Fundación MaratóTV3, tras evaluación a cargo de la Agencia Nacional Española de Evaluación y Prospectiva).

Recibido: 4/08/2009; aceptado: 25/10/2009

Rev. Asoc. Esp. Neuropsiq., 2010; 30 (106), 193-218. 
ORIGINALES Y REVISIONES

\section{Introducción ${ }^{2}$}

Un problema aún abierto en el estudio de los trastornos psicóticos sigue siendo el de su etiopatogenia y sus factores de riesgo. Desde los inicios del siglo $\mathrm{XX}$, numerosos investigadores insistieron en la psicógenesis de la esquizofrenia, bien desde el punto de vista de la psicopatología individual y su psicodinamia (1-11), como desde los estudios e intervenciones familiares (12-13). Al mismo tiempo, desde el paradigma biológico, Rudin y Kraepelin plantearon el origen genético de la esquizofrenia (14). Por otro lado, los primeros estudios sobre la epidemiología de los trastornos mentales, aportaron datos que apoyaban la importancia de los factores sociales en el desarrollo de los trastornos psicóticos y las esquizofrenias, así como acerca de su acumulación en colectivos humanos con menor nivel socioeconómico: los estudios de Faris y Dunham (15), Hollinshead y Redlich (16), Roger Bastide (17) o Raymond Cochrane (18), han de considerarse pioneros en este ámbito.

Sin embargo, actualmente, la hipótesis genética de la esquizofrenia sigue siendo considerada habitualmente como la hipótesis explicativa fundamental, bien sea en su versión más dura, más geneticista $(14,19,20)$, bien sea en la perspectiva de su introducción en las hipótesis de la diátesis-estrés o de la vulnerabilidad (21-27). Así, desde una perspectiva biológico-genética, la esquizofrenia estaría "causada" o "determinada" por alteraciones genéticas que ponen en marcha microlesiones estáticas del encéfalo, o bien alteraciones en el neurodesarrollo que son las determinantes de tal patología $(22,26)$. Parece que hoy deberíamos concebir el trastorno esquizofrénico como vinculado con lesiones encefálicas estáticas (cerebrales y cerebelosas) que comprometen sobre todo al sistema límbico, al tálamo y a sus conexiones con el cortex prefrontal dorsolateral: los síntomas "en negativo" se asociarían con la disfunción del córtex PDL y los síntomas "en positivo", con la disfunción del sistema límbico y la hiperfunción DA mesolímbica. De esa forma, el inicio de los síntomas de la esquizofrenia no depende del tiempo de inicio de la lesión, que podría incluso postularse como intrauterino o en los primeros momentos de la vida extrauterina, sino que se vincula con los momentos en los cuales las zonas cerebrales afectadas alcanzan la madurez fisiológica mediante la puesta en marcha de trasformaciones neurales genéticamente determinadas -por ejemplo, los cambios neuro-endocrino-inmunitarios y psicosociales que se conocen como "pubertad" y "adolescencia". Así, la esquizofrenia estaría determinada por cambios o factores de riesgo que ponen en marcha bien sea microlesiones estáticas del en-

\footnotetext{
${ }^{2}$ Parte de los datos empíricos de este trabajo han sido publicados inicialmente en Tizón, JL et al., Neighborhood differences in psychoses: Prevalence of psychotic disorders in two socially-differentiated metropolitan areas of Barcelona. Schizophrenia Research (2009) ;112(1-3): 143-148, doi:10.1016/j.schres.2009.04.008.
} 
ORIGINALES Y REVISIONES

céfalo, bien sea las alteraciones en el neurodesarrollo que son las determinantes de tal patología (21-27).

En consecuencia, los estudios sobre la génesis y la genética de la esquizofrenia han adquirido hoy en día una enorme complejidad y dificultad $(14,19,28-35)$. Por un lado, porque desde la perspectiva de la genómica, el trastorno ha revelado su indudable base poligénica y multifactorial: Las revisiones sobre el tema hablan de una evidencia convergente, proveniente de los estudios de "linkage", apuntando hacia determinadas áreas del genoma humano (5q, 6p, 6q, 8p, 10p, 13q, 18p y 22q) las cuales podrían contener el material genético que contribuyen como etiología -aunque tal vez habría que decir como "factor de riesgo" genético-- al desarrollo de la esquizofrenia. Sin embargo "las señales replicadas de linkage son débiles" (20) y el progreso en el Proyecto del Genoma Humano y en los estudios de familias, junto con estrategias clínicas apoyadas en factores de riesgo y protección selectivos y bien definidos, tendrán que prestar una ayuda, probablemente decisiva, para la investigación del material genómico susceptible.

El campo de los estudios basados en la genética familiar, los estudios con gemelos mono y dicigóticos han supuesto un ámbito de trabajo investigador ya antiguo y mantenido $(19,20,30,31)$. Pero, si las técnicas con gemelos son generalmente difíciles, costosas y largas, mucho más lo son en el caso de la esquizofrenia. Nuevamente, por al menos dos razones: Primera, porque los estudios de gemelos, para ser creíbles, han de basarse en similitudes y diferencias entre gemelos monocigóticos y dicigóticos sometidos a separación desde el nacimiento. Ahora bien: no hay que perder de vista que, con esa "separación desde el nacimiento", desde el punto de vista ambientalista, psicológico o psicosocial, se introduce una variable o cúmulo de variables difíciles de controlar y habitualmente mal controladas. A menos que se parta de entrada de suponer demostrada la hipótesis genética que se intenta demostrar, separar a un gemelo de su hermano y su familia, desde el nacimiento, supone una grave situación traumática para toda la familia, sostenida a lo largo de años, y que, cómo no, puede afectar al gemelo de múltiples formas. El trabajo de Tienari et al (31) es a menudo considerado como un hito en este terreno, como unos años antes lo fue el de Kendler y Robinetti (30): Tienari et al. siguieron durante décadas a más de 200 niños finlandeses adoptados cuya madre padecía esquizofrenia, y los compararon con otros niños adoptivos cuyos padres no la padecían. Se aplicaron controles y reglas de anonimato y se realizaron mediciones del funcionamiento de la familia de adopción. El riesgo de padecer esquizofrenia se encontró que era más alto en los individuos cuyos progenitores la padecían, apoyando los estudios que hablan de la contribución genética a la esquizofrenia. Sin embargo, ese aumento de la incidencia debido a factores supuestamente genómicos, sólo se halló en las familias cuyo funcionamiento se había diagnosticado como perturbado. Ello pose al 
menos dos implicaciones: 1) El ambiente familiar matiza la expresión genética y 2) Existen "factores protectores" o "de contención” en determinadas familias que valdría la pena reconocer de cara a la prevención y el tratamiento (35).

Pero las técnicas con gemelos son especialmente difíciles, costosas y largas, en el caso del "síndrome esquizofrénico" porque, en segundo lugar, para realizar estudios sobre la genética de la esquizofrenia, previamente hay que diagnosticar tal trastorno y su incidencia diferencial en diversos tipos de poblaciones. Y aquí, como sucedía en los estudios sobre genética familiar, acaecen importantes problemas. Antes que nada, el de las tasas de incidencia y prevalencia del trastorno, ampliamente diferentes aún según la metodología empleada, según las perspectivas teóricas de los profesionales y según las poblaciones estudiadas. Resúmenes de esa problemática aparecen en la bibliografía de este trabajo (36-62) y han sido tratadas por nosotros en trabajos anteriores $(28,29,63,64)$. El segundo problema, y no menor, es el de fiabilidad del mismo diagnóstico de "esquizofrenia" y, más allá, el de la validez de tal grupo diagnóstico (65).

En nuestra perspectiva, la hiper-valorización creciente de los datos biológicos sobre la etiología de los trastornos esquizofrénicos puede estarnos llevando a una simplificación abusiva del campo estudiado. Una simplificación que no tiene suficientemente en cuenta tanto la heterogeneidad actual de los datos, como la posible heterogeneidad etiológica del síndrome que describen. Y todo ello, sin menospreciar la posibilidad de que, como afirman algunos investigadores $(5,33,34)$, el síndrome esquizofrénico no sea más que un síndrome que el futuro de la investigación tenderá a diversificar en varias "enfermedades" y/o "trastornos" diferenciados. En último extremo con los datos actualmente existentes no es disparatado pensar que, lo que hoy llamamos "esquizofrenia": 1) No es más que un síndrome fundamentalmente descrito tres un acuerdo entre expertos seleccionados. 2) Es factible pensar que pueda ser el resultado de un desarrollo psicopatológico de larga evolución. 3) Que, si no es cuidado adecuadamente en sus primeros años de evolución, da lugar a esas psicosis desintegrativas postpuberales, en ocasiones de larga evolución. 4) Y son éstas las que se han tenido en cuenta para elaborar los criterios de esquizofrenia", tanto en el DSM como en las CIE de la OMS (66).

La tendencia hacia la uniformización mediante el reduccionismo biologista puede observarse en cómo, aceptando la hipótesis de la diátesis-estrés o la de los trastornos en el neurodesarrollo y la vulnerabilidad de Weinberger (21-29), tienden a simplificarse las "causas" de la esquizofrenia en las (supuestas) alteraciones genéticas, en microlesiones encefálicas, o bien en trastornos en los neurotransmisores: esos son los estudios, investigaciones y sistematizaciones dominantes en la literatura científica sobre el tema $(20,59)$. Los "factores psicosociales" aparecen habitualmente como una especie de apéndice. A menudo se 
ORIGINALES Y REVISIONES

afirma que la esquizofrenia es un trastorno genéticamente determinado porque "los familiares biológicos de primer grado de los sujetos con esquizofrenia presentan un riesgo para la esquizofrenia aproximadamente diez veces superior al de la población general ( $i ? ?)$. Las tasas de concordancia para la esquizofrenia son mas altas en los gemelos monocigotos que en los dicigotos....", etc. Luego, las matizaciones a tales afirmaciones que aparecen descritas... pero a pie de página o "en letra pequeña". Es una buena "muestra en estado de transición" de cómo los factores psicológicos individuales y familiares así como los factores sociales tienden a desaparecer de los textos, guías y resúmenes: primero se reducen; luego, pasan a letra pequeña o a pié de página; al final, ¿desaparecerán?.

Y ello a pesar de que la debilidad patente de esa perspectiva no biológica, sino biologista (y, por tanto, disociadora), radica en que no se ha demostrado que esas alteraciones neuronales sean previas y, por tanto, causantes del trastorno. Porque, ¿no podrían ser también una posible consecuencia neuroevolutiva de microlesiones, conflictos, déficits y/o inhibiciones de las primeras fases del desarrollo humano?. ¿O un resultado biológico del impacto de sufrimientos y aislamientos afectivo-sensoriales primigenios graves y precoces $(28,29)$ ?. Tal vez por todo ello, los estudios sobre la genética de la esquizofrenia han adquirido hoy en día esa enorme complejidad y dificultad (19, 20, 30-34).

Teniendo en cuenta esos razonamientos, ya hace años nos parecía que recoger datos sobre epidemiología, características sociales, psicosociales y psicopatológicas de los pacientes psicóticos en la comunidad podría proporcionar matizaciones a esas generalizaciones tal vez demasiado rápidas y simples. En concreto, según nuestra experiencia, los estudios con población general, al menos los clásicos de "doble fase", corren el riesgo de no detectar los casos más crónicos de psicosis, o los menos desadaptados / desequilibrados, que son los que ingresan menos ...(36-40, 43, 49, 56, 57, 60). Por el contrario, los estudios basados en los hospitales, en particular los estudios puntuales o de "cortes transversales", difícilmente detectarán los esquizofrénicos más conservados, más contenidos, con menos recidivas o menos graves... (42, 49, 52, 57, 61, 62). En definitiva, los que no ingresan ${ }^{3}(63,64)$.

Interesaría, pues, realizar estudios del tipo del ABC alemán, por ejemplo $(38,39)$. Pero estudios que incluyan tanto los pacientes más integrados en la comunidad como los habitualmente detectados por su inadaptación: los que realizan ingresos

\footnotetext{
${ }^{3}$ Por ejemplo, de nuestra población de 838 pacientes psicóticos y al menos 4.937 con diagnósticos de "trastornos mentales severos" (1988-2000), ingresaron en un servicio hospitalario durante el año 2000, 136 pacientes. Ello significa que, de una población de pacientes psicóticos definida, ingresaron anualmente menos del $8 \%$. Durante el 2002, nuestra población de 105.531 habitantes (nuevo censo) realizó 611 visitas en los servicios hospitalarios de urgencias de Barcelona (de Hospital Psiquiátrico o General) así como 128 ingresos psiquiátricos.
} 
psiquiátricos o acuden a los dispositivos secundarios y terciarios de psiquiatría y salud mental (38-46). Todo ello equivale a decir que interesa realizar estudios de los pacientes detectados y con registro de casos que viven en la comunidad; es decir, de los pacientes detectados en la Atención Primaria de Salud, en los Servicios Sociales y comunitarios y en la intersección de la Atención Primaria sanitaria y social con la Red de Salud Mental: la línea asistencial o los dispositivos que en otros lugares hemos llamado "atención primaria a la salud mental" (67-69).

En nuestra opinión, la realización de ese tipo de estudios poblacionales, con la inclusión de pacientes con una mayor diversidad evolutiva de sus trastornos psicopatológicos, puede contribuir: 1) A aclarar la etiopatogenia y los factores de riesgo de la esquizofrenia y otras psicosis; 2) A verificar o falsar la hipótesis de que en comunidades socialmente empobrecidas o con menor nivel socioeconómico aumenta la presencia de población con esquizofrenia y otras psicosis con respecto a comunidades con mayor nivel socioeconómico.

Para llevar a cabo una investigación de este tipo, se necesita un equipo profesional estrechamente vinculado con la clínica de la salud mental comunitaria y conectado con la APS. En 1999, nuestro equipo de investigación, vinculado con la Unidad de Salud Mental de Sant Martí-La Mina (USMSMLM), diseñó el proyecto SASPE (Señales de Alarma y Síntomas Prodrómicos de la Esquizofrenia) ${ }^{4}$. Dicho proyecto, a través de diferentes líneas de trabajo, intenta contribuir a la investigación de los trastornos esquizofrénicos y las psicosis delirantes en la comunidad mediante la determinación de su prevalencia y factores de riesgo en la población atendida durante los años 1982-2004 por dicha USM (la población de los barrios colindantes de la Verneda, del distrito de Sant Martí de Barcelona y La Mina, del municipio de Sant Adrià del Besos). El trabajo que aquí se presenta se enmarca dentro de dicho proyecto $(63,64)$, uno de cuyos objetivos era determinar y comparar la prevalencia de esquizofrenia y otras psicosis en la población general y población de riesgo de los barrios de la Verneda y La Mina del área metropolitana de Barcelona.

\footnotetext{
${ }^{4}$ El proyecto SASPE obtuvo en 1999 una beca de investigación de la Fundación Seny, tras la evaluación de los proyectos realizada por la Agencia de Evaluación de la Investigación y las Tecnologías Médicas de Catalunya, organismo vinculado a la OMS. Ayuda a la investigación 02E/99 Fundació Seny-AATM-OMS.
} 
ORIGINALES Y REVISIONES

\section{Población y Métodos del Estudio de Prevalencia SASPE}

Diseño:

Se trata de un estudio descriptivo transversal en una población en tratamiento por una USM (la USM Sant Martí-La Mina, dependiente del Institut Català de la Salut). Dicha USM estaba integrada dentro de los dispositivos sanitarios de la Atención Primaria y especialmente conectada con los equipos de pediatría y medicina de familia.

Sujetos y Fuentes de Información:

La población sobre la que se realizó este estudio corresponde a dos barrios del área metropolitana de Barcelona, el de la Verneda (perteneciente al distrito de Sant Martí, de la ciudad de Barcelona) y el de La Mina (de la ciudad de Sant Adrià del Besos, provincia de Barcelona), separados simplemente por una calle intermedia rudimentariamente urbanizada. Ambas barriadas se incluyen en el área metropolitana de Barcelona. Mientras que la población de la Verneda es mayoritariamente de clase social media y media-baja (grupos II, III y IV del SES), la población del barrio de La Mina se caracteriza por pertenecer mayoritariamente a los grupos IV y V, y por su inadaptación social, su inestabilidad económico-social y una amplia problemática relacionada con la marginalidad (70-72). (Tablas 1 y 2). Sanitariamente, la población total se organiza en cinco Áreas Básicas de Salud (ABS), que en el año 2000 agrupaban en total 103.615 habitantes. Desde hace más de 20 años, estas ABS poseen de referencia a la USM de Sant Martí-La Mina, que funciona dentro de varios Centros de Atención Primaria de la zona.

Para seleccionar los casos de esquizofrenia y otras psicosis se ha utilizado el registro de casos clínicos de la USM, basado en su estilo de trabajo que hemos llamado programa de "Atención primaria a la salud mental" (67-69), cuyo resultado más visible es una mayor accesibilidad del servicio y un trabajo conjunto de los diversos dispositivos pertenecientes a diversos departamentos. Posteriormente, para detectar psicosis, se han seleccionado todos aquellos sujetos que cumplieran los criterios de inclusión previamente definidos por el equipo investigador para el Proyecto SASPE, resumidos en las tablas 3 y 4 . Para este trabajo se han incluido todos los sujetos que cumplieran dichos criterios desde 1982 al año 2000.

En el registro de casos clínicos de la USM se hayan incluidos todos los pacientes que en algún momento han sido visitados en la USM aunque luego, en un pequeño porcentaje de casos, el tratamiento haya sido encomendado a la APS con la colaboración del equipo especializado o bien a otros dispositivos. (en la mayoría de los casos, la USM intentaba trabajar en red con los servicios sociales, sanitarios y pedagógicos y con terapias combinadas, intentado evitar la medicalización y/o la dependencia de la población con respecto al sistema sanitario). Se ha depurado el registro eliminando del mismo todos los casos que, en una com- 
paración con datos del Censo de población del año 2000 o del Padrón de 1996, se halló que habían muerto o se habían trasladado de residencia.

Los datos de población de La Verneda provienen de la actualización del Censo del año 2000 y los de La Mina del Padrón de 1996 ya que, por pertenecer administrativamente a otro municipio, no se hallaban aún disponibles los del 2000, año del cierre de la muestra.

Análisis de datos

Se ha realizado el cálculo de la prevalencia de esquizofrenia y otras psicosis, en población general y en población en edad de riesgo (15-54 años) en ambos barrios y se han calculados los intervalos de confianza de las diferencias. Se ha utilizado para ello el Programa SPSS para Windows en su versión 13.0.

Resultados del Estudio SASPE A acerca de diferencias de prevalencia.

En la tablas 5 y 6 se presenta el diagnóstico detallado de los 838 casos de la USM de Sant Martí diagnosticados de "esquizofrenia y otras psicosis delirantes" entre los años 1982 y 2000. En primer lugar aparece el diagnóstico de esquizofrenia $(48,2 \%)$ seguido por el de trastornos delirantes $(24,9 \%)$.

En la tabla 7, se presentan los datos de prevalencia de esquizofrenia y otras psicosis en población general y población de riesgo. La prevalencia de esquizofrenia en población general era de 45,9 por 10.000 habitantes (I.C.95\% $=41,8$ $50,1)$ y en población de riesgo, de 71,7 (I.C.95\% =65-78,6). La prevalencia de “otras psicosis" era de 34,9 por 10.000 habitantes en población general (I.C.95\% =31,3-38,5), y de 36,1 en población en riesgo (I.C.95\% = 31,3-41,0).

La tabla 8 y la figura 1 muestran la significación de las diferencias en los datos de prevalencia de esquizofrenia y otras psicosis en población general y población de riesgo entre los dos barrios. La prevalencia de trastornos psicóticos totales y en sus diversos apartados en La Mina es superior a la de la Verneda tanto en población general (134,8 por 10.000 habitantes), como en población en edad de riesgo (177,5 por 10.000 habitantes). Puede observarse que el barrio de La Mina posee también una mucha mayor prevalencia de esquizofrenia respecto al barrio de la Verneda tanto en la población general como en la población en riesgo (esquizofrenia en población general en La Mina: 76 por 10.000 habitantes, I.C.95\% de la diferencia de tasas: 27,2-50,9; esquizofrenia en población de riesgo en La Mina: 116,1; I.C.95\% de la diferencia de tasas: 38,9-77,2). 
ORIGINALES Y REVISIONES

\section{Discusión}

Dadas las características y vinculaciones de esta USM con la APS y con los equipos de referencia de segundo y tercer nivel, creemos que nuestro estudio proporciona datos de prevalencia bastante cercanos a los que podrían extraerse de investigaciones con población general. En efecto, como hemos explicitado en otros trabajos $(63,64,67,68)$, la Unidad de Salud Mental de Sant Martí-La Mina (I.C.S.), que proporcionó la base clínica y gran parte de los miembros del equipo de investigación, se distingue por:

a) Ser un equipo estable, de orientación integradora de la perspectiva comunitaria con la perspectiva psicoanalítica y la biológica, que lleva trabajando en el mismo territorio y con la misma población y orientación desde 1982.

b) Está encuadrada dentro de una organización asistencialmente delimitada y estrechamente territorializada de la red de salud mental, APS y servicios sociales, de forma que sólo los pacientes que utilicen únicamente la red privada pueden no ser detectados por dicha unidad.

c) Dicha unidad combina la atención primaria a la salud mental de adultos y de niños: está formada por dos equipos para la atención de adultos, uno de atención específica a los TMS (o trastornos mentales graves), dos para la atención de niños y jóvenes y una "Unidad funcional de atención a la primera infancia", sostenida junto con los pediatras y otros profesionales de atención precoz de la zona. Durante 11 años, además, integró un CAS (Centro de Atención y Seguimiento para drogodependientes).

d) Tiene una de las más altas accesibilidades estudiadas y publicadas de Europa: a finales del 2001, se había visitado en alguna ocasión en la Unidad 22.846 personas de la población adscrita (103.615 personas), lo que corresponde al 22'05 por ciento de la población de su territorio.

e) En consecuencia, todo el equipo está vitalmente interesado en programas de prevención y promoción de la salud mental (67-69), dada la inadecuación de profesionalizar a una proporción de la población tan alta.

f) Al ser la población y el territorio delimitados, la accesibilidad tan amplia y la clase social media-baja, baja y marginal (grupos IV y V), es difícil que un porcentaje importante de la población afectada por trastornos mentales severos (TMS) no pase por dicha unidad, aunque sea a buscar las recetas de sus prescripciones, las pruebas complementarias, los ingresos en dispositivos secundarios o terciarios de salud mental... Estimamos que dicho porcentaje es menor del 10 por ciento y que, en el caso de los trastornos psicóticos, no llega al 1 por ciento. Para fundamentar tal estimación podemos aportar el dato complementario de que la prevalencia en servicio de pacientes con diagnósticos catalogables dentro del grupo de los "trastornos mentales graves" según los estándares habituales era en 
el 2001 del 22' 46 por ciento del total de pacientes detectados (5.132 pacientes de esos grupos de diagnósticos).

g) En consecuencia, la tasa de "esquizofrenias" y "otras psicosis" detectados hasta el momento por dicha unidad es alto: según el Estudio SASPE "A", 838 (1982-2000), a los que hay que añadir entre 100 y 150 nuevos pacientes pertenecientes al estudio SASPE B (2001 y 2002) $(63,64,67)$. Tal como esperábamos, si nuestras búsquedas son suficientemente exhaustivas, y la cohorte suficientemente representativa de la realidad, el tipo de "casos" detectados en ella coincidiría más estrechamente con al realidad del trastorno psicótico en la comunidad que otro tipo de estudios y formas de detección.

Esas matizaciones previas contribuyen a valorar la radicalidad de los resultados: Como acabamos de resaltar, el barrio de La Mina, sujeto a numerosos factores de riesgo psicosocial (71-73), posee una prevalencia en servicio para el conjunto de las psicosis de 134,84 por 10.000 habitantes en población general; en cambio, en el de La Verneda con unos indicadores socioeconómicos más favorables, esas prevalencias eran de 64,90. Para la esquizofrenia en concreto, las tasas son de 76 y 116 por 10.000 (La Mina) y 37 y 58 por 10.000 (La Verneda), según se considere en cada caso la población general o la población en edad de riesgo. Es decir, ciertas tasas de psicosis en La Mina eran hasta 2 veces mayores que las encontradas en la Verneda por el mismo equipo y en el mismo período temporal.

En comparación con otros estudios, los resultados de prevalencia de nuestra investigación se sitúan entre los más elevados, sobre todo los de La Mina, máxime si tenemos en cuenta que estamos hablando de un estudio de prevalencia en servicio. Así, según el National Institute of Mental Health norteamericano, a partir del estudio ECA $(36,43,44)$, la prevalencia anual de la esquizofrenia oscilaba entre 0'9 y 1'3 por cien. El estudio español de Vázquez-Barquero en Cantabria (España) $(57,58,62)$ aportaba una prevalencia de esquizofrenia y otras psicosis entre 0,5 y 1,1 por cien. En el estudio de Navarra (España) (37) la prevalencia anual se situaba alrededor del 0 ' $8 \%$ y la prevalencia asistida alrededor del 0 '2\%. Puede observarse las variaciones existentes entre los diferentes trabajos, las cuales en parte son debidas a diferentes ámbitos y metodología de los estudios (poblacional, hospitalario, etc): En sí mismas, esas variaciones deberían reforzar la necesidad de cambiar los enfoques de la investigación poblacional y clínica y, tal vez, de la necesidad de plantearnos la validez del diagnóstico (y el concepto) de esquizofrenia $(65,66)$.

Un dato destacado de nuestro estudio, al que le dedicaremos un trabajo específico, es la alta proporción de pacientes con trastorno delirante diagnosticados, mucho mayor que en otros trabajos, lo cual probablemente se halle en relación con la alta accesibilidad de la USM estudiada. El dato posee una relevancia clínica, poblacional y social que es preciso profundizar y perfilar. 
ORIGINALES Y REVISIONES

Los datos aportados por esta investigación sugieren de nuevo la importancia de los factores sociales y psicosociales en la perpetuación ("prevalencia") y, tal vez, en la génesis de "las esquizofrenias" y, en general, de las psicosis. Así, el barrio de la La Mina con unos peores indicadores psicosociales y socioeconómicos (71-73), presenta una prevalencia de trastornos psicóticos en general y en especial de "esquizofrenia" muy superiores a los de la Verneda. Estas diferencias encontradas, no pueden atribuirse a una distinta atención sanitaria entre ambos barrios ya que las dos poblaciones son atendidas por los profesionales pertenecientes a la misma USM los cuales utilizan en ambos barrios idénticos protocolos de actuación sanitaria.

De la influencia de los factores sociológicos y psicosociales nos proporcionan datos estudios como los de Tienari (31), Alanen (35) y Castilla (74), Allardyce y Boydell $(54)$, Van Os $(55,56)$, Olin et al $(98,99)$ y otros muchos. Asimismo, la realidad clínica cotidiana nos muestra la radical importancia que para el futuro de un esquizofrénico joven, posee el que, durante el primer episodio del trastorno, sea tratado de forma "antipsicológica" o bien mediante el tratamiento combinado que hoy se sabe que es imprescindible en estos casos (35, 75-79, 84-87). Pero es que en numerosos medios asistenciales no suele utilizarse aún ese tratamiento mixto --o incluso no siempre se incluye en determinados textos de "Terapéutica" (valdría decir entonces, de "terapéutica biologistamente reducida") $(66,67,69)$

Desgraciadamente, tanto a nivel popular como a nivel de los grupos de investigación nos vamos acostumbrando a que en la psiquiatría dominante en nuestro medio predomine una fuerte tendencia a sobrevalorar la importancia de los factores de riesgo biológicos. O, más aún, a considerarlos causas (y causas determinantes) $(28,29)$ de toda la psicopatología -y, más aún de las psicosis. Así, como antes recordábamos, se llega a decir que la esquizofrenia es un trastorno genéticamente determinado porque "los familiares biológicos de primer grado de los sujetos con esquizofrenia presentan un riesgo para la esquizofrenia aproximadamente diez veces superior al de la población general" (59). Pero, a menudo, en nuestro medio se olvidan las matizaciones a tales hallazgos que aparecen descritas... por ejemplo, en las líneas siguientes de la misma obra colectiva que estamos citando 5 .

Así, para algunos autores, "La esquizofrenia es probablemente un trastorno poligénico de penetrancia incompleta"... Y "la secuencia ontogenética en una

\footnotetext{
5 Que dice a continuación: "Los estudios de adopción han demostrado que los familiares biológicos de los sujetos con esquizofrenia tienen un riesgo sustancialmente elevado para desarrollar esquizofrenia, mientras que los familiares adoptivos no tienen aumentado el riesgo. A pesar de que numerosos datos sugieren la importancia de los factores genéticos en la etiología de la esquizofrenia, la existencia de una discordancia sustancial de la frecuencia en gemelos monocigotos también indica la importancia de los factores ambientales" (59).
} 
enfermedad (como la esquizofrenia) puede ser fundamental para explicar su base orgánica" (80). Pero, como recordábamos hace poco $(67,79)$, los datos acerca de factores de riesgo en las relaciones precoces y en las relaciones familiares para la génesis de la esquizofrenia son hoy ya importantes en cantidad, y al menos tan llamativos como los que apoyan la patogenia biológica del síndrome $(53,54)$. Además, en las psicoterapias individuales o familiares de pacientes esquizofrénicos, estamos acostumbrados a encontrarnos con elementos que nos confirman nuestra perspectiva de la existencia de una psicodinamia del deterioro y la mejoría psicótica... Pero también, con la existencia de núcleos y defensas, como las simbiótico-adhesivas (81-83), muy difícilmente alterables o cambiables mediante cualquier tipo de tratamiento (y que determinados tratamientos psicológicos o farmacológicos pueden incluso empeorar). Eso nos obliga a plantearnos una y otra vez la realidad de los determinantes biológicos y psicológicos del síndrome.

En este mismo ámbito de la evolución del trastorno esquizofrénico, los más viejos de entre nosotros conocimos "los otros esquizofrénicos", los esquizofrénicos previos a la extensión de los nuevos psicofármacos y al movimiento desinstitucionalizador impulsado inicialmente por el psicoanálisis, la antipsiquiatría y la psiquiatría comunitaria... A pesar de los desvaríos, errores y excesos teóricos y prácticos de tales movimientos y de las prácticas comunitarias y des-institucionalizadoras posteriores a la "anti-psiquiatría", la "historia natural" de la esquizofrenia ha cambiado tanto, con sus innegables claro-obscuros actuales, que hay que valorar la importancia de los factores sociales $(66,74) \ldots$ Factores, por cierto, remarcados por el hecho de que el pronóstico de la esquizofrenia a los dos y cinco años en Agra (India), Cali (Colombia) e Ibadan (Nigeria) es significativamente mejor que en los países tecnológicamente "desarrollados" $(42,47)$. En algún lugar de las profundas diferencias en alojamiento, dieta, cuidados maternos, triangulaciones originarias, cuidados microsociales, educación, sistemas de trabajo, exposiciones al trastorno, creencias, costumbres y demás contingencias habrá que buscar las variables que expliquen esas diferencias... (53, 54, 83, 88101).. Por ejemplo, cada vez hay más datos que hacen pensar en la relevancia de los factores psicológicos (aislamiento, conflictos graves y crónicos, procesos de duelo cronificados...) o biológicos (hambrunas, virasis, otras enfermedades...) sufridos por las madres embarazadas en la primera parte de su embarazo, en los momentos de la formación del SNC del nuevo ser (100), como factores de riesgo para las psicosis de esos hijos en gestación.

Posibles limitaciones de nuestro estudio empírico: Algunos de los factores biopsicosociales que tal vez sesguen nuestros datos e interpretaciones podrían ser los siguientes:

1) En primer lugar, la posibilidad de que La Mina fuera en esos años un barrio de "deriva social", es decir, un barrio en el cual se concentren las personas 
ORIGINALES Y REVISIONES

marginales, marginalizadas, desclasadas, etc. Se trata de habitats en los cuales la investigación, desde Faris y Dunham (15) o Cochrane (18), ha detectado un aumento de psicopatología y, en particular, de trastornos mentales graves. Pero, ya desde su fundación, La Mina no era un barrio “de aluvión”. En realidad, durante los años del estudio su población ha sido tan o más estable que la de los barrios circundantes, tanto según las estadísticas municipales como en los estudios realizados al respecto (71-73). La "deriva social" no parece jugar aquí un papel fundamental, como no lo jugaba la llegada de inmigrantes de países no pertenecientes a la Unión Europea, aún muy reducida en los años estudiados (246 sobre una población total de 23.657 habitantes según el padrón municipal), en coincidencia con las observaciones clínicas realizadas, tanto en la USM como en los dispositivos de APS.

2) Otra posible fuente de sesgos es la numerosa población de etnia gitana del barrio, que se estima en el 33\% (en las barridas circundantes, entre el 5 y el $17 \%$ según el padrón municipal). Si la etnia gitana tuviera una carga genética de psicosis o esquizofrenia mayor, ello podría haber influido en nuestros resultados de forma importante. Se han llevado a cabo búsquedas bibliográficas, pero no hemos encontrado estudios relevantes que demostrasen esa mayor "carga genética" de esquizofrenia en esa etnia, ni siquiera de un aumento de la incidencia o prevalencia de la esquizofrenia entre los gitanos (102). Más bien la opinión de algunos clínicos que trabajan en estos medios apunta a una menor incidencia de la psicosis entre estos habitantes y a una mayor atención familiar y social a estos pacientes dentro de la etnia. En nuestro caso, cualquier dato habría que matizarlo, además, debido a que una buena parte de los gitanos de La Mina se hallan en una situación de anomia y des-estructuración cultural sumamente importante, en parte por causa de las influencias desorganizadoras de las drogas ilegales y el propio tráfico de drogas. Es evidente que en esta situación pesan mucho más los elementos sociales y psicosociales que los genéticos.

3) Existiría la posibilidad de que, por la amplitud numérica de las familias gitanas, pudieran haberse concentrado en nuestro estudio varios "cluster" familiares genéticamente muy incisivos, en especial si no se dan situaciones favorables en el medio social y en otros niveles para la contención $(28,29,31,35,68$, 70). Dada la concentración de los apellidos gitanos, que a menudo pertenecen más a clanes que a familias en el sentido "payo" del término, sólo el análisis pormenorizado de las historias de los 319 casos detectados en La Mina permitiría delimitar la influencia del factor familiar. Este trabajo de revisión ya ha sido puesto en marcha por el equipo investigador, y los primeros resultados obtenidos, basándose en los apellidos de los pacientes, ponen en duda la importancia de tal influencia en nuestra muestra, aunque no es un elemento que haya que marginar precipitadamente en una valoración cuidadosa del conjunto de factores. 
4) Otra argumentación de corte biológico, aunque no genética, que aparentemente limitaría el valor de nuestros datos, es el importante número de afecciones somáticas de los habitantes de La Mina (tabla 1) y, por tanto, la alta probabilidad de que las madres sufran enfermedades intercurrentes en el embarazo y la primera infancia de los hijos, lo cual conllevaría factores de riesgo para padecer la esquizofrenia más adelante $(53,54,84-101)$. En nuestra opinión, la importancia relativa que los estudios realizados conceden a esos factores no explicaría las diferencias halladas, aunque es un argumento no desdeñable. Sin embargo, no olvidemos que se trataría de una repercusión a nivel biopsicológico de factores de riesgo sociales, que son los que estamos intentado re-valorizar.

5) Una posibilidad para pensar en desviaciones en los resultados obtenidos, vendría dada por los sesgos intrínsecos a los clínicos que trabajan con poblaciones marginales, desfavorecidas, o de etnia o clase social no concordante con la del explorador: tienden a calificar a los consultantes más frecuentemente con diagnósticos del tipo de "psicosis" o "trastornos mentales severos". El tema fue estudiado entre nosotros hace años para el ámbito de las migraciones (103) y está siendo retomado, por ejemplo, en los estudios sobre comunicación y salud del grupo de la Universidad de Rochester (104). Se trata de otro argumento no desdeñable, pero que hemos intentado controlar de entrada con los criterios de selección de "caso" para el estudio SASPE, los cuales implican siempre más de un acto diagnóstico y la realidad de que dichos pacientes están siendo tratados por esas afecciones y no sólo "han sido diagnosticados" como afectados por las mismas.

A pesar de las matizaciones anteriores, parece que la explicación más plausible de las diferencias en la prevalencia de la esquizofrenia halladas entre los dos barrios estudiados, vendría proporcionada por los factores sociales y psicosociales, posiblemente a través de su acción sobre las capacidades de contención de las familias, grupos e instituciones sociales. Nuestros datos apoyan la necesidad de tener en cuenta la importancia de los factores de riesgo sociales y psicosociales en la génesis y prevalencia de la esquizofrenia y las psicosis. También apuntan el peligro que supondría una exclusión o infravaloración de dichos factores: y no sólo para la clínica, sino para toda la concepción de dichos trastornos. La importancia del tema bien merece que otros equipos investigadores realicen estudios similares en barrios marginales y semi-marginales, así como de comparación entre poblaciones social y psicosocialmente diferenciadas, con el fin de validar o perfilar los resultados de nuestro estudio. 
ORIGINALES Y REVISIONES

Tabla 1

Características y Factores de Riesgo de la población del barrio de "La Mina", en la metrópoli de Barcelona, en los años del estudio (datos elaborados por JL Tizón desde la Dirección de la Unidad de Salud Mental de Sant Martí-La Mina).

\begin{tabular}{|c|c|}
\hline Característica & $\begin{array}{l}\text { Estimación sobre } \\
\text { la población total }\end{array}$ \\
\hline Población & $18.657-23.657^{*}$ \\
\hline Indice de embarazos en adolescentes & $\begin{array}{l}>\text { que en población general, } \\
\text { pero indeterminado }\end{array}$ \\
\hline Incidencia de psicopatología infanto-juvenil detectada en servicio en un año. & $1 ’ 7 \%$ \\
\hline Incidencia de psicopatología en adultos detectada en servicio en un año & $1{ }^{\prime} 02 \%$ \\
\hline Porcentaje de la población visitada en algún momento por la Unidad de Salud mental. & $>20 \%$ \\
\hline $\begin{array}{l}\text { Drogodependencias detectadas } \\
\text { - ADVP } 350 \\
\text { - Metadona } 160 \\
\text { • Otras drogas } 500 \\
\text { - TOTAL } 1.010\end{array}$ & $439 \%$ \\
\hline Personas con SIDA y HIV + detectadas: $\mathrm{N}=180$ & $0 ’ 78 \%$ \\
\hline $\begin{array}{l}\text { Etnias } \\
\qquad \begin{array}{l}\text { - Caucásicos } \\
\text { - Etnia gitana: } \\
\text { - Otras etnias determinadas } \\
\text { - No determinados }\end{array}\end{array}$ & $\begin{array}{l}63,5 \% \\
33 \% \\
0 ’ 9 \% \\
2{ }^{\prime} 6 \%\end{array}$ \\
\hline Analfabetos detectados : 2.562 & $14{ }^{\prime} 32 \%$ \\
\hline Jubilados: 3.964 & $16 \cdot 75 \%$ \\
\hline Pensionistas e inválidos: 5.407 & $22{ }^{\prime} 85$ \\
\hline $\begin{array}{l}\text { Alto índice de enfermedades crónicas: } \\
\text { • HTA: } 2.620 \\
\text { • Dislipemias: } 2.110 \\
\text { • Diabetes: } 1229 \\
\text { - EPOC: } 950 \\
\text { - Enfermedades reumatológicas....>1000 casos }\end{array}$ & $>25 \%$ \\
\hline Inmigrantes extracomunitarios: 246 & $\begin{array}{l}\text { 13'18 sobre la población } \\
\text { censada }\end{array}$ \\
\hline Media de Hijos por familia & 2'6 (Barcelona: 1'7) \\
\hline Media de Personas por familia & 5'6 (Barcelona: 3’3) \\
\hline
\end{tabular}

* Población censada / Población estimada al alza utilizada por los administradores sanitarios del sector para incluir numerosas personas no censadas. 
ORIGINALES Y REVISIONES

Tabla 2

ESQUEMA DE LOS PROBLEMAS DEL BARRIO desde el punto de vista social y psicosocial en los años del estudio (datos elaborados por JL Tizón desde la Dirección de la Unidad de Salud Mental de Sant Martí-La Mina) (71-73).

1. Inestabilidad FAMILIAR:

Deterioro importante en las relaciones de pareja y en las relaciones padres-hijos: abandonos o semi-abandonos, separaciones, incapacidad de la madre, el padre o ambos para atender a los hijos por causas mentales, pobreza y condiciones económicas extremas, malos tratos, drogodependencia con deterioro familiar grave, alcoholismos, trastornos mentales en los padres

---> Desestabilización de los niños y adolescentes.

2. Inestabilidad CULTURAL:

Anomia importante en las dos culturas dominantes, con las consecutivas actitudes antisolidarias, redes delictivas y de "protección", chauvinismo, aislamiento cultural y social, inhibición ante la promoción y la formación... (en especial, de las mujeres).

3. Inestabilidad ECONÓMICA:

Alto índice de paro, actividades económicas marginales o delictivas, numerosos pensionistas y jubilados con pensiones mínimas, impago moroso de alquileres, transacciones ilegales de viviendas, ocupaciones ilegales de viviendas, uso ilegal de las redes públicas de agua, gas y electricidad...

4. Inestabilidad URBANÍSTICA:

Continuos "planes de remodelación" y continuos rumores de "demolición" del barrio.

\section{DELINCUENCIA:}

Tráfico de drogas, compra-venta de oro y otros objetos, tráfico de armas, "protección" de establecimientos mediante amenazas, ambiente urbano deteriorado, especialmente por la noche...

\section{Problemas de la INFANCIA Y LA ADOLESCENCIA:}

Absentismo escolar (especialmente de las niñas y jóvenes), inadaptación social y cultural, actividades marginales y delincuenciales, internamientos en instituciones por deficiencias familiares, deficiencias mentales y físicas más frecuentes, alteraciones familiares y en la capacidad de cuidado y contención de los padres, condiciones socioeconómicas extremas, abandonos totales o parciales por parte de la madre y/o el padre... 


\section{ORIGINALES Y REVISIONES}

Tabla 3

Diagnósticos DSM-IV utilizados para el diagnóstico de "caso" en el Proyecto SASPE

(Señales de Alerta y Signos Prodrómicos de la Esquizofrenia).

\begin{tabular}{|l|l|}
\hline Esquizofrenia & $\begin{array}{l}295.10,295.20,295.30, \\
295.60,295.90\end{array}$ \\
\hline Trastorno esquizofreniforme & 295.40 \\
\hline Trastorno esquizoafectivo & 295.70 \\
\hline Trastorno delirante & 207. * $^{*}$ \\
\hline Trastorno psicótico breve & 298.8 \\
\hline Trastorno psicótico compartido & 297.3 \\
\hline Trastorno psicótico debido a enfermedad médica & $293 . * *$ \\
\hline Trastorno psicótico inducido por sustancias & 292.11 \\
\hline Trastorno psicótico no especificado & $298.9 *$ \\
\hline Trastornos psicóticos en la infancia: trastornos generalizados del desarrollo. & $299.00,299.10,299.80$ \\
\hline
\end{tabular}

Tabla 4

Criterios para el diagnóstico de "caso" en el Proyecto SASPE.

\begin{tabular}{|l|l|}
\hline Criterios & \\
\hline A. & $\begin{array}{l}\text { A1. Los diagnósticos de la tabla 3 realizados según criterios DSM-IV. } \\
\text { A2. Mantenidos un mínimo de 6 meses. } \\
\text { A3. Y en tres o más visitas en la Unidad de Salud Mental. }\end{array}$ \\
\hline B. & $\begin{array}{l}\text { O bien: } \\
\text { B. Dos o más ingresos en Servicios de Psiquiatría Hospitalarios o de Hospitales monográficos } \\
\text { con esos grupos diagnósticos (tabla 3). }\end{array}$ \\
\hline C. & $\begin{array}{l}\text { O bien: } \\
\text { C. Dos o más visitas en Urgencias de Hospital General o Servicio de Psiquiatría de Hospital } \\
\text { con esos diagnósticos de salida (tabla 3). }\end{array}$ \\
\hline
\end{tabular}


Tabla 5

Diagnósticos detallados de los casos de "Esquizofrenia" y "Otras Psicosis" diagnosticados en la Unidad de Salud Mental de Sant Marti-La Mina. Barcelona, 1982-2000.

\begin{tabular}{|c|c|c|}
\hline Diagnóstico DSM-IV & Frecuencia & Porcentaje (\%) \\
\hline - Esquizofrenia & 404 & $48 ’ 2$ \\
\hline - Trastorno Esquizofreniforme & 38 & $4 ' 5$ \\
\hline - Trastorno Esquizoafectivo & 34 & 4 '1 \\
\hline - Trastorno delirante. & 209 & $24{ }^{\prime} 9$ \\
\hline - Trastorno psicótico breve & 11 & $1 ' 3$ \\
\hline - Trastorno pscótico compartido & 1 & $0 ’ 1$ \\
\hline - Trastorno psicótico debido a enfermedad médica. & 9 & 1 '1 \\
\hline - Trastorno psicótico inducido por substancias & 10 & $1 ’ 2$ \\
\hline - Trastorno psicótico no especificado & 62 & $7 ’ 4$ \\
\hline $\begin{array}{l}\text { - Trastornos psicóticos en la infancia (0-14 años). } \\
\text { Diagnósticos DSM IV: } 299.00,299.10 \text { y } 299.80\end{array}$ & 60 & $7 ’ 2$ \\
\hline Totales & 838 & $100 ’ 0$ \\
\hline
\end{tabular}

Tabla 6

Diagnósticos detallados de los casos de "Esquizofrenia" y "Otras Psicosis" en la población general y en la población de riesgo en los dos barrios. Muestra: 1982-2000.

\begin{tabular}{|c|c|c|c|c|c|c|}
\hline & \multicolumn{3}{|c|}{ Población General } & \multicolumn{3}{|c|}{ Población de riesgo (15-54 años) } \\
\hline & La Verneda & La Mina & Total & La Verneda & La Mina & Total \\
\hline $\mathrm{N}$ & 79958 & 23657 & 103615 & 45071 & 13859 & 58930 \\
\hline N 0-14 años & 9211 & 3993 & 13204 & & & \\
\hline N Trastornos psicóticos & 518 & 318 & 838 & 393 & 243 & 636 \\
\hline $\mathrm{N}$ "Esquizofrenia"* & 296 & 180 & 476 & 262 & 161 & 423 \\
\hline N Otras psicosis & 223 & 139 & 362 & 131 & 82 & 213 \\
\hline $\mathrm{N}$ Trastornos delirantes & 145 & 64 & 209 & 99 & 45 & 144 \\
\hline N Otras psicosis en la edad adulta $* *$ & 47 & 46 & 93 & 32 & 37 & 69 \\
\hline N Psicosis en la infancia (0-14 años) & 31 & 29 & 60 & & & \\
\hline
\end{tabular}

* Incluye los casos de esquizofrenia, trastornos esquizofreniformes y trastornos esquizoafectivos.

** Incluye los trastornos psicóticos breves, compartidos, no especificados, y los debidos a enfermedades médicas o inducidos por abuso de substancias. 
ORIGINALES Y REVISIONES

Tabla 7

Prevalencia en población general y en edad de riesgo del diagnóstico de "esquizofrenia" y "otras psicosis" en la Unidad de Salud Mental de Sant Martí-La Mina (Barcelona): Muestra 1982-2000.

\begin{tabular}{|c|c|c|c|c|c|c|c|c|}
\hline & \multicolumn{4}{|c|}{ Población General ( $\mathrm{n}=103.615)$} & \multicolumn{4}{|c|}{ Población en edad de riesgo $(\mathrm{n}=58.930)^{* * *}$} \\
\hline & $\mathbf{N}$ & $\%$ & $\begin{array}{c}\text { Prevalecencia } \\
\text { x } 10.000\end{array}$ & I.C. $95 \%$ & $\mathbf{N}$ & $\%$ & $\begin{array}{c}\text { Prevalecencia } \\
\text { x } 10.000\end{array}$ & I.C. $95 \%$ \\
\hline Esquizofrenia * & 476 & 56,8 & 45,93 & $41,8-50,1$ & 423 & 66,5 & 71,78 & $65-78,6$ \\
\hline "Otras Psicosis" & 362 & 43,2 & 34,93 & $31,3-38,5$ & 213 & 33,5 & 36,14 & $31,3-41$ \\
\hline - Trastornos delirantes & 209 & 24,94 & 20,17 & $17,4-22,9$ & 144 & 22,6 & 24,43 & $20,4-28,4$ \\
\hline $\begin{array}{l}\text { - Otras psicosis } \\
\text { del adulto } * *\end{array}$ & 93 & 11,1 & 8,97 & $7,1-10,8$ & 69 & 10,9 & 11,7 & $8,9-14,5$ \\
\hline $\begin{array}{l}\text { - Otras psicosis } \\
\text { de inicio en la infancia } \\
(0-14 \text { años })(n=13.204)\end{array}$ & 60 & 7,16 & 45,44 & $34-56,9$ & & & & \\
\hline Totales & 838 & 100 & $\mathbf{8 0 , 8 7}$ & $75,4-86,3$ & 636 & 100 & $107,9 * * * *$ & $99,6-116$ \\
\hline
\end{tabular}

* Incluye los casos de esquizofrenia, los trastornos esquizofreniformes y los trastornos esquizoafectivos.

** Incluye los trastornos psicóticos breves, los trastornos psicóticos compartidos, los trastornos psicóticos debidos a enfermedad médica, los trastornos psicóticos inducidos por substancias y los trastornos psicóticos no especificados.

*** Población en Edad de Riesgo, $15-54$ años $=58.930$

**** Se excluyen los casos de "otras psicosis de inicio en la infancia". 
Tabla 8

Diferencias en la prevalencia del diagnóstico de "esquizofrenia" y "otras psicosis" en el barrio de La Verneda y en el de La Mina de la Unidad de Salud Mental de Sant Marti-La Mina (Barcelona). Muestra: 1982-2000.

\begin{tabular}{|c|c|c|c|c|c|}
\hline & \multicolumn{5}{|c|}{ Población General } \\
\hline & \multicolumn{2}{|c|}{ La Verneda (3) } & \multicolumn{2}{|c|}{ La Mina (4) } & \multirow[b]{2}{*}{$\begin{array}{c}\text { Diferencia de } \\
\text { prevalencias I.C. } 95 \%\end{array}$} \\
\hline & $\mathbf{N}$ & $\begin{array}{c}\text { Prevalecencia } \\
\text { x } 10.000\end{array}$ & $\mathbf{N}$ & $\begin{array}{c}\text { Prevalecencia } \\
\text { x } 10.000\end{array}$ & \\
\hline Esquizofrenia (1) & 296 & 37,01 & 180 & 76 & $39,1(27,2-50,9)$ \\
\hline Otras Psicosis & 223 & 27,88 & 139 & $\mathbf{5 8 , 7}$ & $30,9(20,5-41,3)$ \\
\hline - Trastornos delirantes & 145 & 18,13 & 64 & 27 & $8,9(1,6-16,2)$ \\
\hline $\begin{array}{l}\cdot \text { Otras psicosis } \\
\text { del adulto (2) }\end{array}$ & 47 & 5,87 & 46 & 19,4 & $13,6(7,7-19,4)$ \\
\hline $\begin{array}{l}\text { - Otras psicosis } \\
\text { de inicio en la infancia } \\
(0-14 \text { años })(8) \text { y (9) }\end{array}$ & 31 & 33,6 & 29 & 72,6 & $39(10,1-67,8)$ \\
\hline Totales & 519 & 64,9 & 319 & 134,8 & $69,9(54,2-85,7)$ \\
\hline
\end{tabular}

\begin{tabular}{|c|c|c|c|c|c|}
\hline & \multicolumn{5}{|c|}{ Población en edad de riesgo } \\
\hline & \multicolumn{2}{|c|}{ La Verneda (5) } & \multicolumn{2}{|c|}{ La Mina (6) } & \multirow[b]{2}{*}{$\begin{array}{c}\text { Diferencia de } \\
\text { prevalencias I.C. } 95 \%\end{array}$} \\
\hline & $\mathbf{N}$ & $\begin{array}{c}\text { Prevalecencia } \\
\text { x } 10.000\end{array}$ & $\mathbf{N}$ & $\begin{array}{c}\text { Prevalecencia } \\
\text { x } 10.000\end{array}$ & \\
\hline Esquizofrenia (1) & 262 & 58,1 & 161 & 116,1 & $58(38,9-77,2)$ \\
\hline Otras Psicosis & 133 & 29,5 & 85 & 61,3 & $31,8(17,9-45,8)$ \\
\hline - Trastornos delirantes & 99 & 21,9 & 45 & 32,4 & $10,5(0,09-20,9)$ \\
\hline $\begin{array}{l}\text { - Otras psicosis } \\
\text { del adulto (2) }\end{array}$ & 32 & 7 & 37 & 26,6 & $19,6(10,7-28,5)$ \\
\hline \multicolumn{6}{|l|}{$\begin{array}{l}\text { - Otras psicosis } \\
\text { de inicio en la infancia } \\
(0-14 \text { años })(8) \text { y (9) }\end{array}$} \\
\hline Totales & 395 & $87,6(7)$ & 246 & $177,5(7)$ & $89,9(66,3-113)$ \\
\hline
\end{tabular}

(1) incluye los casos de esquizofrenia, los trastornos esquizofreniformes y los trastornos esquizoafectivos.

(2) incluye los trastornos psicóticos breves, los trastornos psicóticos compartidos, los trastornos psicóticos debidos a enfermedad médica, los trastornos psicóticos inducidos por substancias y los trastornos psicóticos no especificados.

(3) Población General La Verneda $=79.958$

(4) Población General La Mina $=23.657$

(5) Población en Edad de Riesgo, 15-54 años, La Verneda $=45.071$

(6) Población en Edad de Riesgo, 15-54 años, La Mina = 13.859

(7) Se excluyen los casos de "otras psicosis de inicio en la infancia".

(8) Población de 0-14 años, La Verneda $=9.211$

(9) Población de 0-14 años, La Mina = 3.993 
ORIGINALES Y REVISIONES

Figura 1

Diferencias en la prevalencia del diagnóstico de "esquizofrenia" y "otras psicosis" en el barrio de La Verneda y en el de La Mina de la Unidad de Salud Mental de Sant Marti-La Mina (Barcelona).

Muestra: 1982-2000. (N por diez mil)

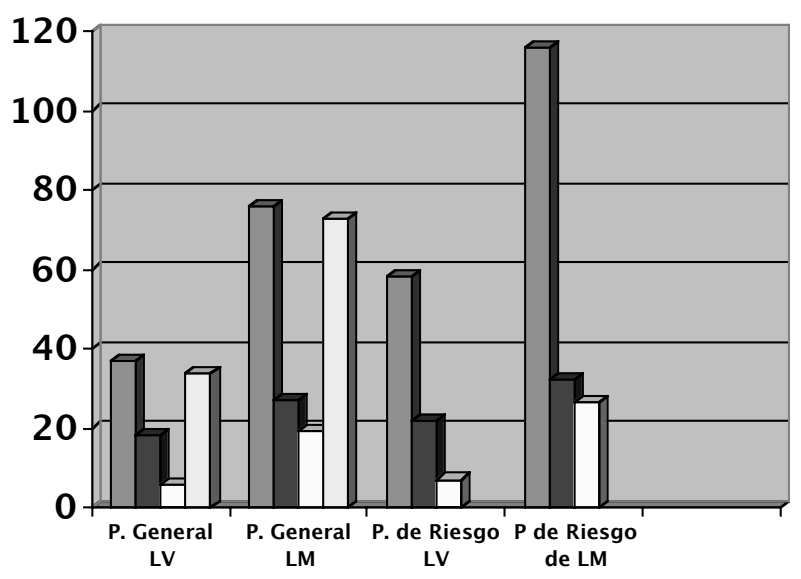

\begin{tabular}{|l|}
\hline$\square$ "Esquizofrenia" \\
$\square$ Trastornos \\
delirantes \\
Otras Psicosis \\
del adulto \\
$\square$ Psicosis en la \\
infancia
\end{tabular}

V

LM

LV

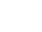




\section{BIBLIOGRAFÍA:}

(1) FreUd S. Puntualizaciones psicoanalíticas sobre un caso de paranoia ("Dementia Paranoides") descrito autobiográficamente (caso "Schreber"). Obras Completas, XII (pp. 11-73). Buenos Aires: Amorrortu 1989.

(2) FreUd S. La pérdida de la realidad en la psicosis y la neurosis. Obras Completas 19. (pp. 191-199) Buenos Aires: Amorrortu 1989.

(3) Klein, M. Notas sobre algunos mecanismos esquizoides. En Obras Completas vol 3 (pp. 10-33). Barcelona: Paidós 1980.

(4) Bleuler E. Dementia Praecox or the Group of Schizophrenias. New York: Int. Universities Press 1952

(5) BellaK L Schizophrenia. New York: Logos Press 1958.

(6) BION WR Differentiation of the psychotic form non-psychotic personalities. En Second Thoughts: Selected Papers on Psycho-Analysis. New York: Jason Aronson 1967, pp 43-64.

(7) Rosenfeld HA. Psychotic States. London: Hogarth Press, 1965.

(8) Freeman, Th. Psicopatología de las Psicosis. Barcelona: Toray, 1972.

(9) Segal, H. A Psychoanalytical Approach to the Treatment of Psychoses. En The Work of H. Segal London: Maresfield Library 1986.

(10) Meltzer D. Metapsicología ampliada. Buenos Aires: Spatia 1999.

(11) Selvini-Palazzoli M, Boscolo L, Ceccin G, Prata G. Paradoja y contraparadoja. Buenos Aires: Paidós 1991.

(12) Minuchin S. Families and Familiy Therapy. Harvard: Harvard University Press 1974

(13) SELVINI M. El futuro de la psicoterapia: Aprender de los errores. Revista de la Asoc. Española de Neuropsiquiatría. 2002, 82: 9-23.

(14) Rieder RO, Kaufman Ch.A Genética. En JA Talbott, RE Hales, SC Yudofsky (dir). Tratado de Psiquiatría. Barcelona: Ancora1989.

(15) Faris REL \& Dunham HW. Mental disorders in urban areas: An echological stiduy of schizophrenia and other psychosis. Chicago: University of Chicago Press 1939.

(16) Hollingshead AB, Redlich FC. Social class and mental illness. New York: John Wiley 1958.

(17) BASTIDE R. Sociologie des maladies mentales. Paris: Flammarion 1965.

(18) Cochrane R. La creación social de la enfermedad mental. Buenos Aires: Nueva Visión 1983.

(19) Maier W, Shwab S, Rietschel M. The genetics of schizophrenia. Current Opinion in Psychiatry 2000,13: 3-9.

(20) KLÄNING U. Greater occurrence of schizophrenia in dizygotic but not monozygotic twins. Registred-based study. Br. J. Psychiatry 1999; 175: 407-409.

(21) WEINBERGER DR. Schizophrenia as a neurodevelopmental disorder: a review of concept. En SR Hirsch, DR Weinberger (eds). Schizophrenia. London: Blackwood 1995.

(22) WeINBERGER DR. From neuropathology to neurodevelopment. Lancet 1995; 346: 552-557.

(23) Weinberger DR, Nasrallah HA. Handbok of Schizophrenia. The Neurology of Schizophrenia. Amsterdam: Elsevier 1986.

(24) Allin M, MurRay R. Schizophrenia: a neurodevelopmental or neurodegenerative disorder?. Current Opinion in Psychiatry 2002, 15: 9-15. 
ORIGINALES Y REVISIONES

(25) ANDREASEN NC. Anomalías cognitivas en la Esquizofrenia. Barcelona: Almirall Prodesfarma 2002.

(26) PARELLADA E. Neurodesarrollo frente a neurodegeneración: hipótesis neuroanatómicas de la esquizofrenia. Psiquiatría Biológica 2001; 6: 225-230.

(27) FRITH CD (ed). The cognitive neurophysiology of schizophrenia. Hover: Lawrence Erlbaum As. 1992.

(28) Tzzón JL. Determinación y determinismo en psicopatología: I. ¿Un problema filosófico?. Archivos de Neurobiología 1999; 62 (4): 277- 289.

(29) Tzzón JL. Determinación y determinismo en psicopatología: II. Una reflexión acerca de los diversos tipos de factores de riesgo. Archivos de Neurobiología 2000; 63, 1: 21-42.

(30) KendLeR KS, Robinette CD: Schizophrenia in the National Academy of Sciences-National Research Council Twin Registry: a 16-year update. Am J. Psychiat 1983,140: 1551-1563.

(31) Tienari P, Wynne LC, Sorri A, Lahti I, Läksy K, Moring J, NaArala M, Nieminen P, WahlBERG KE. Genotype-environment interaction in schizophrenia-spectrum disorder. Long-term follow-up study of Finnish adoptees. Br J Psychiatry. 2004 Mar;184:216-22.

(32) Muller-Hill B. Murderous Science. Oxford: Oxford Univ. Press 1988.

(33) Colodrón A. Las esquizofrenias: "Síndrome de Kraepelin-Bleuler". Madrid: Siglo XXI; 1990.

(34) Овіоls JE. El fin de la esquizofrenia: Por una nueva nosología de las psicosis endógenas. Psiquiatría biológica 2000; 7(6):249-253.

(35) AlAnEN YO. Schizophrenia: Its Origins and Need-Adapted Treatment. London: Karnack Books; 1997, 1999.

(36) REGIER DA, FARMER ME, RAE DS. One-month prevalence of mental disorders in the United States and sociodemographic characteristics: The Epidemiologic Catchement Area Study. Acta Psychiat Scan 1993; 88: 35-47.

(37) Retolaza A. Epidemiología de los trastornos mentales en Atención Primaria. En VázquezBarquero JL (ed.) Psiquiatría en Atención Primaria. Madrid: Aula Médica; 1998 (pp. 55-73).

(38) LASTRA I. El diagnóstico en los estudios epidemiológicos sobre la esquizofrenia. Informaciones Psiquiátricas 2002; 168: 205-216.

(39) HÄFNER H, DER HeIDEN W. Epidemiology of schizophrenia. Can J Psychiatry, 1997;42,2: 139-151.

(40) Häfner H, der Heiden W, Behrens S, Gattaz WF, Hambrecht M, Löffler W, Maurer K, MunkJorgensen P, Nowotny B, Riecher Rössler A, Stein A. Causes and Consequences of the Gender Difference in Age at Onset of Schizophrenia. Schizophrenia Bull, 1998; 24, 1: 99-113

(41) Becker T, Knapp M, Knudsen HC, Schene A, Tansella M, Thornicorft G, Vázquez-BarQUERo JL AND EPSILON STUdy Group. The EPSILON study of shizophrenia in five European countries. Br J Psychiatry 1999, 175: 514-521.

(42) JABLENSKI A. Prevalence and incidence of schizophrenia spectrum disorders: implications for prevention. Aus New Zealand J Psychiatry 2000; 34: S26-S34.

(43) Jones P, CANnon M. The new epidemiology of schizophrenia. Psychiatr Clin North Am, $1998 ; 21,1: 1-25$.

(44) Narrow WE, Rae DS, Robins LN, Regier DA. Revised Prevalence Estimates of Mental Disorders in the United States. Using a Clinical Significance Criterion to Reconcile 2 Surveys' Estimates. Arch Gen Psychiatry 2002; 59: 115-123.

(45) Woods SW, Ziedonis DM, Sernyak MJ, Díaz E, Rosenheck RA. Characteristics of Partici- 
pants and Non-participants in medication Trials for Treatment of Schizophrenia. Psychiat. Services 2000; 51: 79-85.

(46) U.S. Department of Health and Human Services. Mental Health: A Report of the Surgeon General. Rockville MD: National Institute of Mental Health ;1999.

(47) Left JP, Sartorius N, Jablensky A, Kroten A, Ernberg G. The International Pilot Study of Schizophrenia: Five year follow-up findings. Psychol. Med 1992;22: 131-145.

(48) Kendler KS, Gallagher TJ, Abelson JM, Kessler RC. Lifetime prevalence, demographic risk factors, and diagnostic validity of nonaffective psychosis as assessed in a US Community sample. The National Comorbidity Survey. Arch Gen Psychiatry, 1996; 53,11:1022-1031.

(49) Cuesta MJ, Herrera S, Lastra I, Vázquez-Barquero JL. Los factores psicosociales de la esquizofrenia. En F. Rodríguez, JL Vázquez, M Desviat (dirs). Psiquiatría social y Comunitaria. Las Palmas: Instituto Canario de Estudios y Promoción Social y Sanitaria; 1997.

(50) Marcelis M, Navarro Mateu F, Murray R, Selten JP, Van Os J. Urbanization and psychosis: a study of 1942-1978 birth cohorts in The Netherlands. Psychol Med, 1998; 28,4: 871-879.

(51) SALOKANGAS RK. Living situation social network and outcome in schizophrenia: a five-year prospective follow-up study. Acta Psychiatr Scand, 1997; 96,6:459-468.

(52) Torrey EF, Bowler AE, Clark K. Urban birth and residence as risk factors for psychoses: an analysis of 1880 data. Schizophr Res 1997;25,(3): 169-176.

(53) Verdoux H, van Os J, Sham P, Jones P, Gllvarry K, Murray R. Does familiarity predispose to both emergence and persistence of psychosis? A Follow-up study. Br J Psychiatry, 1996; 168,5: 620-626.

(54) Allardyce, J., Boydell, J. Environment and schizophrenia: review: The wider social environment and schizophrenia. Schizophr Bull. 2006;(32) 592-598.

(55) Van Os J, Jones P, Sham P, Bebbington P, Murray RM. Risk factors onset and persistence of psychosis. Soc. Psychiatry Epidemiol, 1998; 33,12: 596-605

(56) VAN Os J. Social influences on risk for disorder and natural history. Current Opinion in Psychiatry 2000; 13:209-213.

(57) Vázquez-Barquero Jl, Díez JF, Muñoz J, Menéndez J, Gaite L, Herrera S, Der GL. Sex differences in mental illness: A community study of the influence of physical health and sociodemographic factors. Soc Psychiatry Epidemiol 1992; 27: 62-68.

(58) Vázquez-Barquero Jl, Cuesta MJ, de la Varga M, Herrera S, Gaite L, Arenal L. The Cantabria first episode schizophrenia study: a summary of general findings. Acta Psychiat Scand 1995; 91 : 156-162.

(59) American Psychiatric Association. DSM-IV: Diagnostic Criteria from DSM-IV. Washington: APA 1994.

(60) Herrera R, Autonell J, Spagnolo E, Doménech J, Martín S. Utilización del método epidemiológico en el estudio de los trastornos mentales. Informaciones Psiquiátricas 1987; 107: 12-20.

(61) Vázquez-Barquero Jl, Lastra I, Cuesta MJ, Herrera S, Gaite L, Pardo G. Análisis psicopatológica de las fases iniciales de la esquizofrenia: Estudio de Primeros Episodios de Esquizofrenia de Cantabria. Psiquiatría Biológica 1995; 2: 138-150

(62) VÁzquez-Barquero JL (ed.) Psiquiatría en Atención Primaria. Madrid: Aula Médica 1998.

(63) Tizón, J.L., Artigue, J., Parra, B., Gomá, M., Ferrando, J., Pareja, F., Sorribes, M., Pérez, C., PARÉs, A. La esquizofrenia en atención primaria: El Estudio SASPE (Señales de alerta y síntomas prodrómicos de la esquizofrenia en APS). Aten Primaria, 2004; (34) :493-498.

(64) Tizón, J.L., Ferrando, J., Parés, A., Artigue, J., Parra, B., Pérez, C., Los trastornos esqui- 
ORIGINALES Y REVISIONES

zofrénicos en la atención primaria a la salud mental. Aten Primaria, 2007; (39): 61-67.

(65) Nordgaard J, Arnfred SM, Handest P, Parnas J: The Diagnostic Status of First-Rank Symptoms.Schizophr Bul. 2008;34(1):137-54. Epub 2007 Jun 11.

(66) Tzzón JL. Los cuidados democráticos de las psicosis como indicador de democracia social. Atopos: Salud mental, comunidad y cultura. 2007; 6: 31-48.

(67) Tzzón JL. Editorial: Resiliencia y contención en pacientes diagnosticados de esquizofrenia. Archivos de Psiquiatría 2006;69(2): 81-84.

(68) Tizón JL. La atención primaria a la salud mental (APSM): Una concreción de la atención sanitaria centrada en el consultante. Atención Primaria 2000; 26,2: 111-119.

(69) Tzzón JL. Una aproximación a las posibilidades de la atención primaria a la salud mental de la infancia. Cuadernos de Gestión 2001;7 (1): 9-30.

(70) Tizón JL. San-José J, Nadal D. Protocolos y programas elementales para la atención primaria a la salud mental. Barcelona: Herder, 1997,2000.

(71) Dal Cin A, De Mesones JL, Tızón JL. Marginal Quarters and Mental Health: A proposition of therapeutic planning of "La Mina", Barcelona, Spain. Case Study Awarded. En Congress Book of 29th ISOCARP Congress, Plenary Sessions. Glasgow: UNESCO - International Society of City and Regional Planners (ISOCARP)- Association Internationale des Urbanistes (AIU) and Internationale Gesselschaft der Stadt.und Regionalplanner (IGSRP); 1993.

(72) DalCin A, de Mesones J, Tzzón JL. La Mina district of Barcelona: A planning approach to psychopathology. Cities 1995; 12,2: 87-95.

(73) Consorci de la Mina, Pla de Transformació del barri de La Mina. Consorci de la Mina, Barcelona; 2007 (también en www.barrimina.org/cat/index.php?option=com_wrapper\&Itemid=46).

(74) Castilla C. El delirio, un error necesario. Oviedo: Nobel;1998.

(75) McGlashan TH. Early detection and intervention in schizophrenia: research. Schizophr Bull, 1996; 22,2:327-345.

(76) McGorry PD, Edwards J, Mihalopoulos C. The EPPIC: A evolving system of early detection and optimal management. Schizophrenia Bulletin. 1996; 22: 305-326.

(77) McGorry P, Edwars J (edITORs). Early Psychoses Training Pack. Chesire, UK: GardinerCaldwell Communications Ltd. 1997.

(78) McGorry PD, Krstev H, Harrigan S. Early detection and treatment delay: Implications of outcome in early psychosis. Current Opinion in Psychiatry 2000; 13: 37-43.

(79) Tzzón JL. Bases para un equipo de atención precoz a los pacientes con psicosis: clínica e investigación pueden aunarse. Revista de la Asoc. Española de Neuropsiquiatría 2009,103(29): 35-62.

(80) Ayuso JL y Salvador S. Manual de Psiquiatría. Madrid: Interamericana-McGraw-Hill; 1992.

(81) Tzzón, JL Psicosis, contención y esperanza: Las funciones emocionales del equipo en el tratamiento de pacientes graves. Informaciones Psiquiátricas 1992;129, 3: 593-616.

(82) Tizón JL. Psicoanálisis, procesos de duelo y psicosis. Barcelona: Herder; 2008.

(83) Fonagy P. The development of Psychopathology form infancy to adulthood: The misterious unfolding of disturbance in time. Plenary Adress at the World Association of Infant Mental Health Congress, Montreal July 28 th 2000.

(84) MAnZANo J (ED.) Las relaciones precoces entre padres e hijos y sus trastornos. Madrid: Necodisne; 2001.

(85) Hingley SM. Psychodynamic perspectives on Psychoses and Psychotherapy: I. Theory. Br.J.Medical Psychology 1997; 70: 301-312. 
(86) Hingley SM. Psychodynamic perspectives on Psychoses and Psychotherapy: II: Practice. Br.J.Medical Psychology 1997; 70: 313-324.

(87) JACKSON M. Weathering the storms: Psychotherapy for psychosis. London: Karnac; 2001.

(88) GatAZ WF, HäFfner H. Search for the Causes of Schizophrenia. Vol IV: Balance of the Century. Berlin: Springer; 1999.

(89) Eisenberg L. The Social Construction of Human Brain. Am J Psychiatry 1995; 152: 15631575.

(90) Eisenberg L Psychiatry and Neuroscience at the end of the century. Curr. Op Psychiat. 1999;12: 629-632.

(91) Institut of Medicine. Reducing Risks for Mental Disorders: Frontiers for Preventive Intervention Research. Washington: The National Academy of Sciences; 2000.

(92) US Department of Health and Human Services. Mental Health: A Report of the Surgeon General. Rockville MD: US Department of Health and Human Service, NIH- NIMH; 1999.

(93) KagAn J, ZeNTNER M. Early childhood predictors of adult psychopathology. Harv Rev Psychiatry. 1996; 3,6: 341-350.

(94) ElLISON Z, van Os J, MuRRay R. Special feacture: childhood personality characteristics of schizophenia: manifestations of, or risk factors for, the disorder?. J. Personal Disord, 1998; 12,3: 247-261.

(95) Isohanni I, Järvelin MR, Nieminen P, Jones P, Rantakallio P, Jokerlaine J, Isohanni M. ScHoOL performance as a predictor of psychiatric hospitalization in adult life. A 28-year follow-up in the Northern Finland 1966 Cohort. Psychol Med., 1998; 28,4:967-974

(96) Jones P, Rodgers B, Murray R, Marmot M. Child developmental risk factors for adult schizophrenia in the British 1946 birth cohort. The Lancet, 1994; 344: 1398-1402.

(97) Jones PB, Rantakallio P, Hartikainen AL, Isohanni M, Siplla P. Schizophrenia as a longterm outcome of pregnancy, delivery, and perinatal complications: a 28-year follow-up of the 1966 north Findalnd general population birth cohort. Am J Psychiatry, 1998;155,3: 355-364.

(98) OLIN SC, Mednick SA. Risk factors of psychosis: identifyng vulnerable populations premorbidly. Schizophr Bull, 1996; 22,2: 223-240.

(99) Olin SC, Mednick SA, Cannon T, Jacobsen B, Parnas J, Schulsinger F, Schulsinger H. School teacher ratings predictive of psichiatric outcome 25 years later. Br J Psichiatry Suppl. 1998; 172,33: 7-13.

(100) Tizón Jl, Buttrago F, Ciurana R, Chocrón L, Fernández MC, García-Campayo J, Montón C, Redondo MJ. Prevención de los trastornos de la salud mental desde la atención primaria de salud. Atención Primaria 2001; 28, supl 2: 96-161.

(101) Khashan AS, Abel KM, McNamee R, Pedersen MG, Webb RT, Baker PN, Kenny LC, Mortensen PB, Higher Risk of Offspring Schizophrenia Following Antenatal Maternal Exposure to Severe Adverse Life Events. Arch Gen Psychiatry. 2008;65(2): 146-152.

(102) FERRER F. El estado de salud del pueblo Gitano en España. Una revisión de la bibliografía. Gaceta Sanitaria 2003;17:2-9.

(103) Tizón JL (COORD.). Migraciones y salud mental. Barcelona: PPU 1983

(104) Epstein RM, Borrell F. Hearing Voices: Patient Centered Care with Diverse Populations. Patient Education and Counseling. 2002;48:1-3. 